\title{
The ALICE time machine
}

\author{
Alessandro Ferretti ${ }^{1}$ \\ ${ }^{1}$ Università di Torino e INFN, sezione di Torino, Via Giuria 1, Torino, Italy
}

\begin{abstract}
According to the Big Bang theory, the Universe was once in an extremely hot and dense state which expanded rapidly. In such a state the normal nuclear matter could not exist: it is believed that a few microsecond after big-bang the matter underwent a phase transition, from a state called Quark-Gluon Plasma (QGP) to a hadron gas. Some of the unexplained features of the Universe could be explained by the QGP properties. One of the aims of the CERN LHC is to recreate (on a smaller scale) a QGP state, compressing and heating ordinary nuclear matter by means of ultrarelativistic heavy-ion collisions. The ALICE experiment at CERN is dedicated to the study of the medium produced in these collisions : in particular, the study of the heavy quarkonia suppression pattern can give a measure of the temperature reached in these collisions, helping us to understand how close we are getting to the conditions of the starting point of the Universe.
\end{abstract}

\section{Early universe and Quark Gluon Plasma}

One of the most accepted theories about the birth of the universe is that everything started almost 14 billion years ago with the so called "Big-Bang": energy and matter were concentrated in a state characterized by an extremely high temperature and energy density.

In those conditions, the state of matter was different from the one that now we are accustomed to. According to QCD lattice calculations, when the temperature of the medium goes above the critical value of temperature $T_{c} \approx 175 \mathrm{MeV}$ (equivalent to $10^{12}{ }^{\circ} \mathrm{K}$ ) and/or over the critical density of $0.3-1$ $\mathrm{GeV} / \mathrm{fm}^{3}$, the color force is no longer strong enough to keep quark and gluons confined into hadrons. A phase transition occurs toward a new state of matter, called Quark Gluon Plasma (QGP), in which the chiral symmetry is restored: more degrees of freedom are available for quark and gluons, which behave as free particles. [1]

The study of the QGP would allow to shed light on many issues: in the framework of this conference, the most interesting one is the quark-to-hadron phase transition that occurred in the early universe, about $10^{-6} \mathrm{~s}$ after the Big Bang. Of course, the challenge is difficult: since (up to now!) it is not possible to travel back in time to see what happened, the alternative is to recreate on a small scale conditions of temperature and energy density similar to the ones of the early universe. How can it be done?

The approach currently used to perform laboratory studies on the QGP is to heat and compress the normal nuclear matter by means of ultra-relativistic collisions between heavy nuclei. The different phases of the evolution of the heavy-ion collision can be summarized as follows: in the first phase (pre-equilibrium phase) deep inelastic scatterings between quark and gluons (partons) of the colliding nuclei occur: these primary collisions lead to the conversion of the center-of-mass energy

This is an Open Access article distributed under the terms of the Creative Commons Attribution License 2.0, which permits unrestricted use, distribution, and reproduction in any medium, provided the original work is properly cited. 
of the nuclei into a number of massive partons, increasing the energy density of the colliding region and leading to the formation of the QGP.

The difference of pressure between the QGP and the surrounding vacuum leads to a hydrodynamical expansion: in the plasma the partons continue to scatter freely until the temperature falls under the critical parameters of temperature and energy density and then hadronizes, freezing the distribution of quark flavours (chemical freeze-out). Hadrons continue to scatter until the thermal freeze-out, when they stop interacting and flow towards the detectors.

Different probes can be exploited in order to collect information about each phase of the evolution. One of the most interesting probes is the heavy quarkonia suppression pattern (measured by means of charmonium and bottonium spectroscopy) which can provide a measure of the temperature reached by the QGP: some details will be given in a following paragraph.

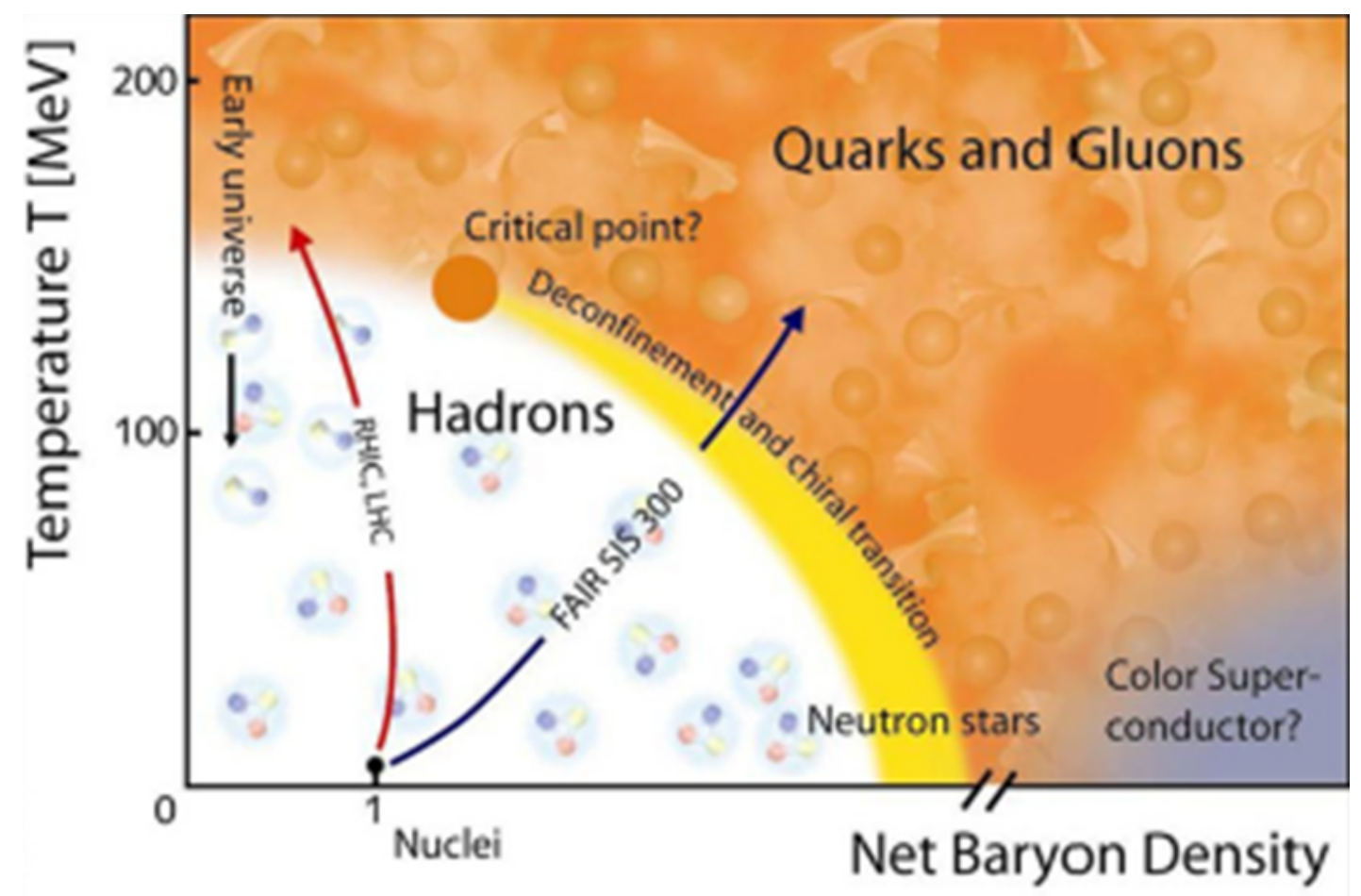

Fig. 1. Phase diagram of the nuclear matter

Since the phase diagram (Fig. 1) of the nuclear matter depends not only on the temperature, but also on the net baryon density (i.e. the baryochemical potential). In the early universe the baryon density was close to zero: since the baryon density of the medium generated in the collision depends on the energy, in order to recreate a situation close to the one of the early state of the Universe the collision energy must be as high as possible, i.e. the energy reachable at the CERN LHC. 


\section{The ALICE experiment}

ALICE (A Large Ion Collider Experiment) is a general-purpose, heavy-ion detector at the CERN LHC [2]. It is designed to address the physics of strongly interacting matter and the QGP at extreme values of energy density and temperature in nucleus-nucleus collisions. It allows a comprehensive study of hadrons, electrons, muons, and photons produced in the collision of heavy nuclei $(\mathrm{Pb}-\mathrm{Pb})$, up to the highest multiplicities anticipated at the LHC. The physics programme also includes collisions with lighter ions and at lower energy, in order to vary energy density and interaction volume, as well as dedicated proton-nucleus runs. Data taking during proton-proton runs at the top LHC energy will provide reference data for the heavy-ion programme and address a number of specific strong-interaction topics for which ALICE is complementary to the other LHC detectors.

ALICE physics goal is to perform a broad set of measurements to address the various interesting topics. A summary of the physics topics and their respective observables is given below:

- Degrees of freedom of quark and gluons as a function of T (observed by means of hadron ratios and spectra, dilepton continuum, direct photons)

- Early state manifestation of collective effects (elliptic flow)

- Energy loss of partons in quark gluon plasma (jet quenching, high pt spectra, open charm and open beauty)

- Study deconfinement (charmonium and bottonium spectroscopy)

- Study chiral symmetry restoration (neutral to charged ratios, resonance decays)

- Detect fluctuation phenomena and critical behavior (event-by-event particle composition, spectra)

- Measure the geometry of the emitting source (HBT, impact parameter via zero-degree energy flow)

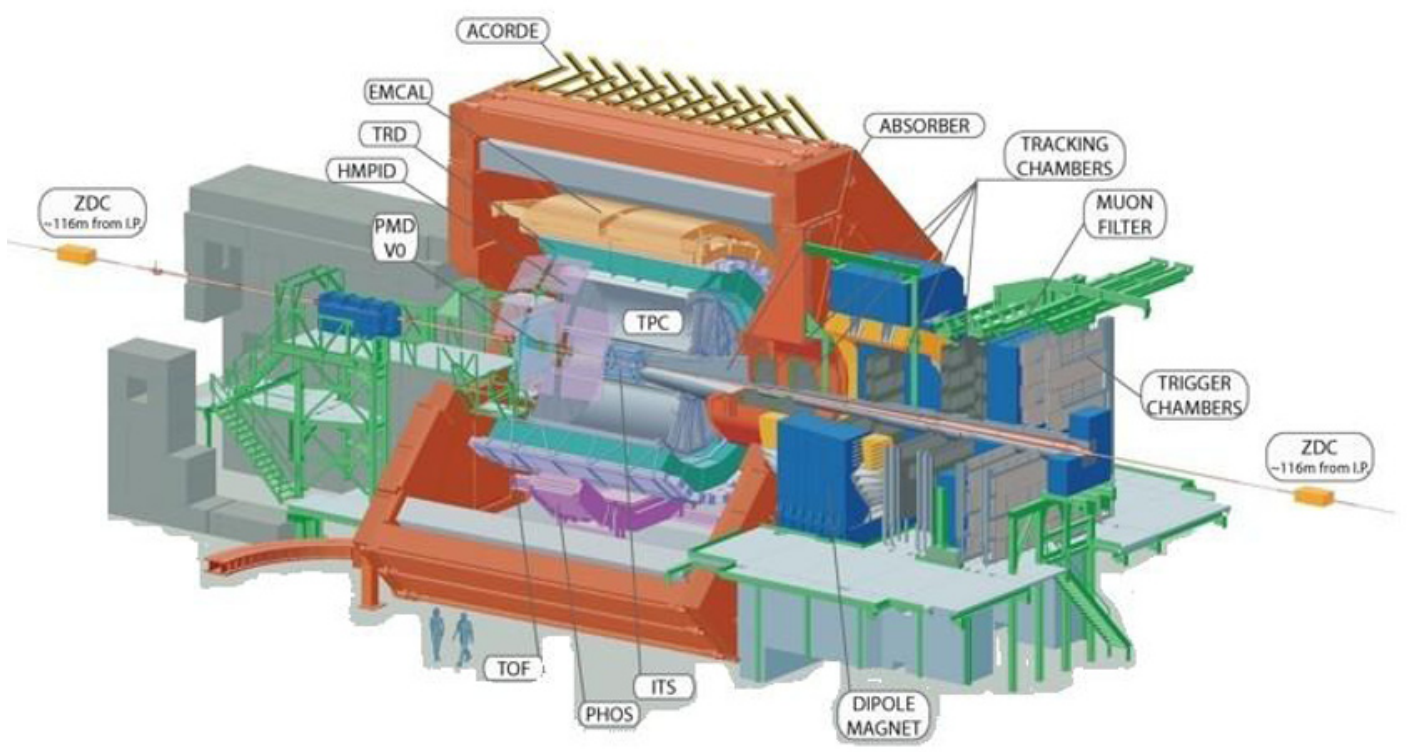

Fig. 2. The layout of the ALICE experiment 
The ALICE detector (Fig. 2) is built by a collaboration including 1200 physicists and engineers from 132 Institutes in 36 countries. Its overall dimensions are $16 \times 16 \times 26 \mathrm{~m}^{3}$ with a total weight of approximately $10000 \mathrm{t}$. ALICE consists of a central barrel part, which measures hadrons, electrons, and photons, and a forward muon spectrometer. The central part covers polar angles from $45^{\circ}$ to $135^{\circ}$ and is embedded in a large solenoid magnet reused from the L3 experiment at LEP. From the inside out, the barrel contains an Inner Tracking System (ITS) of six planes of high-resolution silicon pixel (SPD), drift (SDD), and strip (SSD) detectors, a cylindrical Time-Projection Chamber (TPC), three particle identification arrays of Time-of-Flight (TOF), Ring Imaging Cherenkov (HMPID) and Transition Radiation (TRD) detectors, and two electromagnetic calorimeters (PHOS and EMCal). All detectors except HMPID, PHOS, and EMCal cover the full azimuth. The forward muon arm $\left(2^{\circ}-\right.$ $9^{\circ}$ ) consists of a complex arrangement of absorbers, a large dipole magnet, and fourteen planes of tracking and triggering chambers. Several smaller detectors (ZDC, PMD, FMD, T0, V0) for global event characterization and triggering are located at small angles.

\subsection{Collision centrality determination with ZDCs}

Since heavy nuclei are extended objects the collisions between them can be central or peripheral. The parameter governing the geometry of the collision is the impact parameter $b$, defined as the distance between the trajectories of the colliding nuclei (see Fig. 3). If $b$ is zero the collision is central: when $b$ is larger the collision becomes more and more peripheral. The number of nucleons involved in the primary collisions (participants) decreases increasing $b$, as much as the number of non-interacting nucleons (spectators) increases. The number of participants goes to zero when $\mathrm{b}$ is equal to the sum of the nuclei radiuses (note that if the impact parameter is slightly larger than this value interactions can still occur, such as electromagnetic or diffractive interactions).

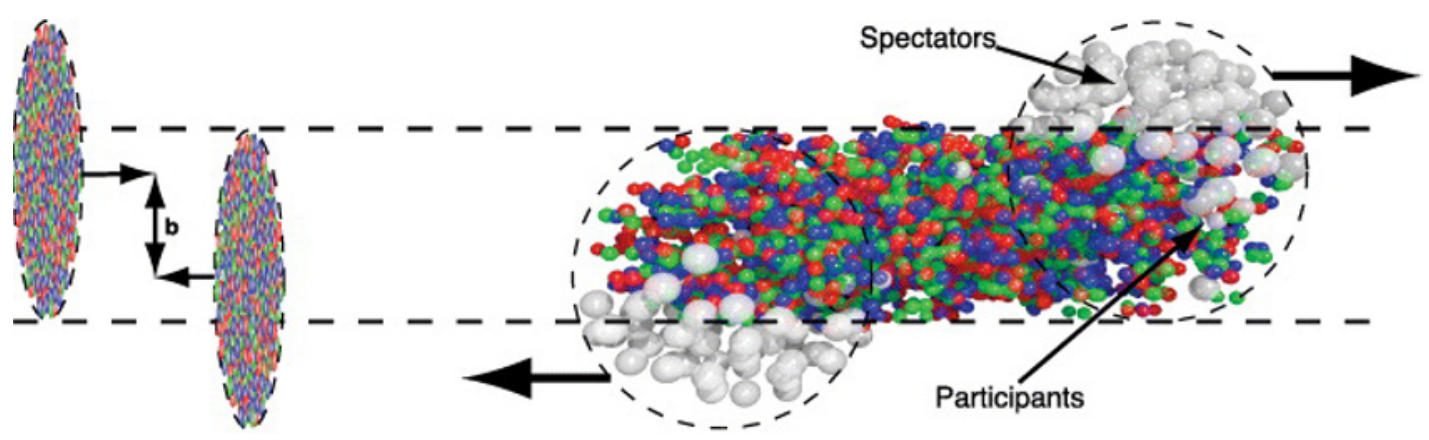

before collision

after collision

Fig. 3. Collision centrality

The geometry of the collisions plays an important role in every possible QGP study: temperature and energy density of the medium created in the interaction depend on the centrality, being maximal for central collisions and decreasing with increasing $b$. Since most of the collisions are peripheral, the measure of centrality is important also for the experiment trigger system, in order to give priority in the DAQ stream to the more rare central collisions.

ALICE Zero Degree Calorimeters (ZDC) [3] have been devised in order to give a fast and reliable measure of the collision centrality. This goal is achieved by measuring the energy carried by the spectator nucleons, which are intercepted by a set of hadronic calorimeters along the beam line 
on both sides of the interaction point (IP) in the zones where the spectators exit the beam pipes, more than $110 \mathrm{~m}$ from IP.

The correlation between the measured energy and the impact parameter is not monotone because when the collision is very peripheral the fraction of nucleus not involved in the collision do not fragment completely. Since larger fragments have a charge-to-mass ratio similar to the one of the original colliding nuclei, LHC magnets do not deviate them out of the beam line enough to be intercepted by ZDCs, and the energy measured is much smaller than expected. To distinguish these events from central ones hadronic ZDC are complemented by a pair of electromagnetic calorimeters (ZEM), placed $7 \mathrm{~m}$ from the IP, in an acceptance window where the energy measured is proportional to the number of primary interactions due to participant nucleons.

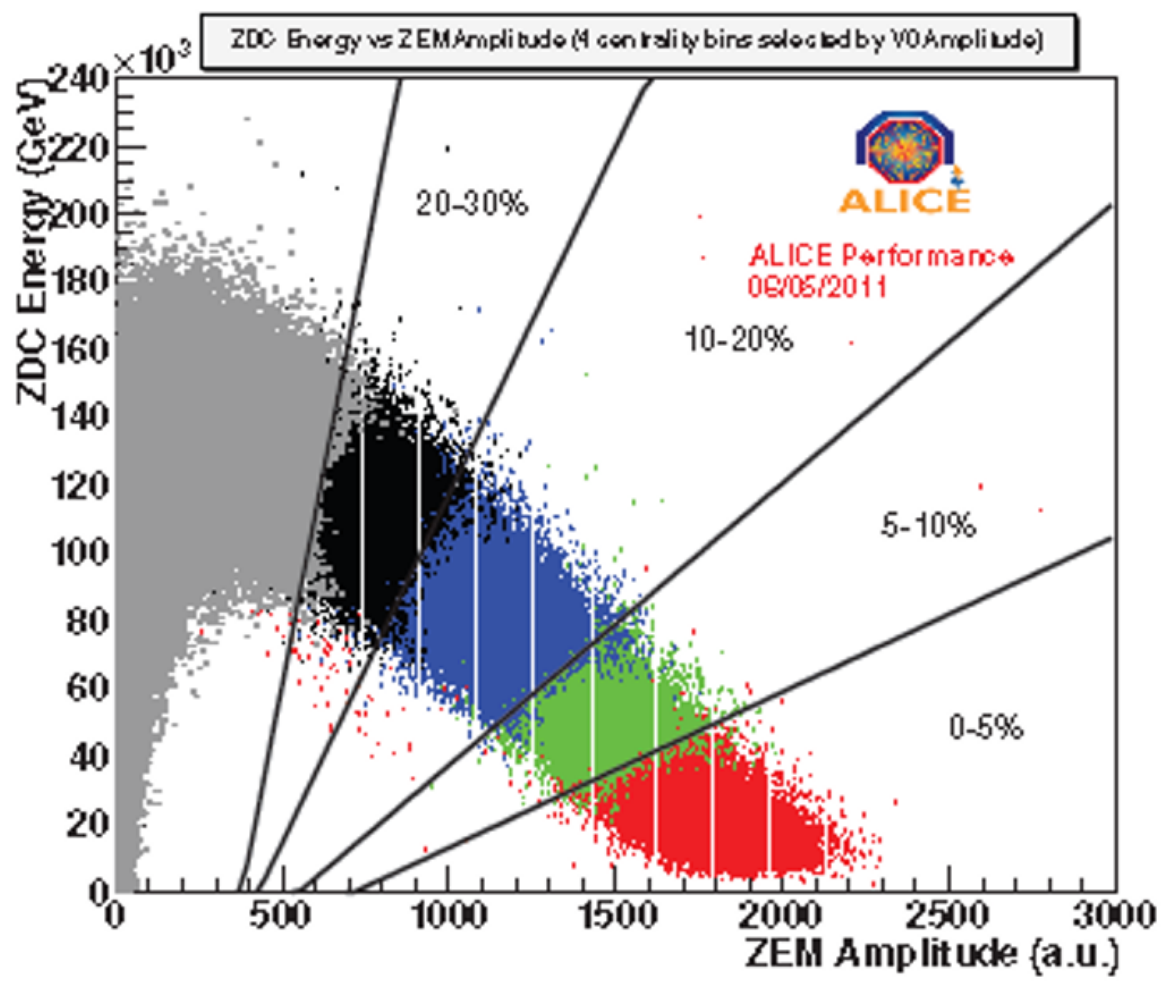

Fig. 4. ZDC centrality classes

ZDCs are sampling calorimeters, with silica optical fibers (which ensure radiation hardness) as active material, embedded in a dense absorber. The spectator nucleons impinging in the absorber create a hadronic shower: the Cherenkov photons emitted by the shower particles traversing the quartz fibers are trapped inside the fiber and collected by the same fiber to a set of photomultipliers. The ZDC system is able to provide a fast trigger signal which is sent to the Central Trigger Processor and allows to select between three tuneable centrality classes, while offline ZDC and ZEM data analysis allows to determine the energy of spectator nucleus with a resolution between $11 \%$ and $13 \%$, i.e. enough to accurately discriminate four centrality classes (5\% most central, $5-10 \%, 10 \%$ $20 \%$ and $20-30 \%$ ) in standalone mode (Fig. 4). 


\section{Heavy quarkonia suppression}

Since 1986, when Matsui and Satz published their seminal work [4], the heavy quarkonia suppression is one of the most active fields of research, both theoretical and experimental, in the quest for QGP. According to QCD lattice calculations, the transition of a dense hadron matter to the QGP state occurs because the colour force which binds together the quarks into hadrons is weakened by the high density of the colour charges, in a way similar to the Debye screening of the electromagnetic force. When the colour charge density is above a certain threshold the colour charge screening leads to the dissolution of bounds that keep hadrons together, giving birth to a deconfined medium.

Heavy quarkonia pairs are interesting because they are formed only in the primary interactions and their survival probability depends on the state of the medium created (deconfined or not) and on its temperature. The bottonium family is formed by the different bound states of a b quark with and anti-b (bbar) partner. The fundamental state, the $Y(1 \mathrm{~S})$, is a very narrow resonance: with a mass of $9.46 \mathrm{MeV}$ it has a width of only $53 \mathrm{keV}$, which means that the b-bbar pair is very strongly bound. If the binding force is screened due to the high density of colour charges, the pair will dissolve. Since the number of b-bbar pairs produced in the initial hard scatterings is relatively low, the probability that the pair will be reformed in the hadronization phase is negligible: the bottonia yield in A-A collision will then be much lower than the yield expected by scaling up an equivalent number of independent nucleon-nucleon collisions. A complementary picture of the situation is given by the fact that in a deconfined medium the number of degrees of freedom of the partons is higher, as well as their energy spectrum. This means that the bottonium can be broken up more easily in a deconfined medium, and the magnitude of suppression will increase with its temperature.

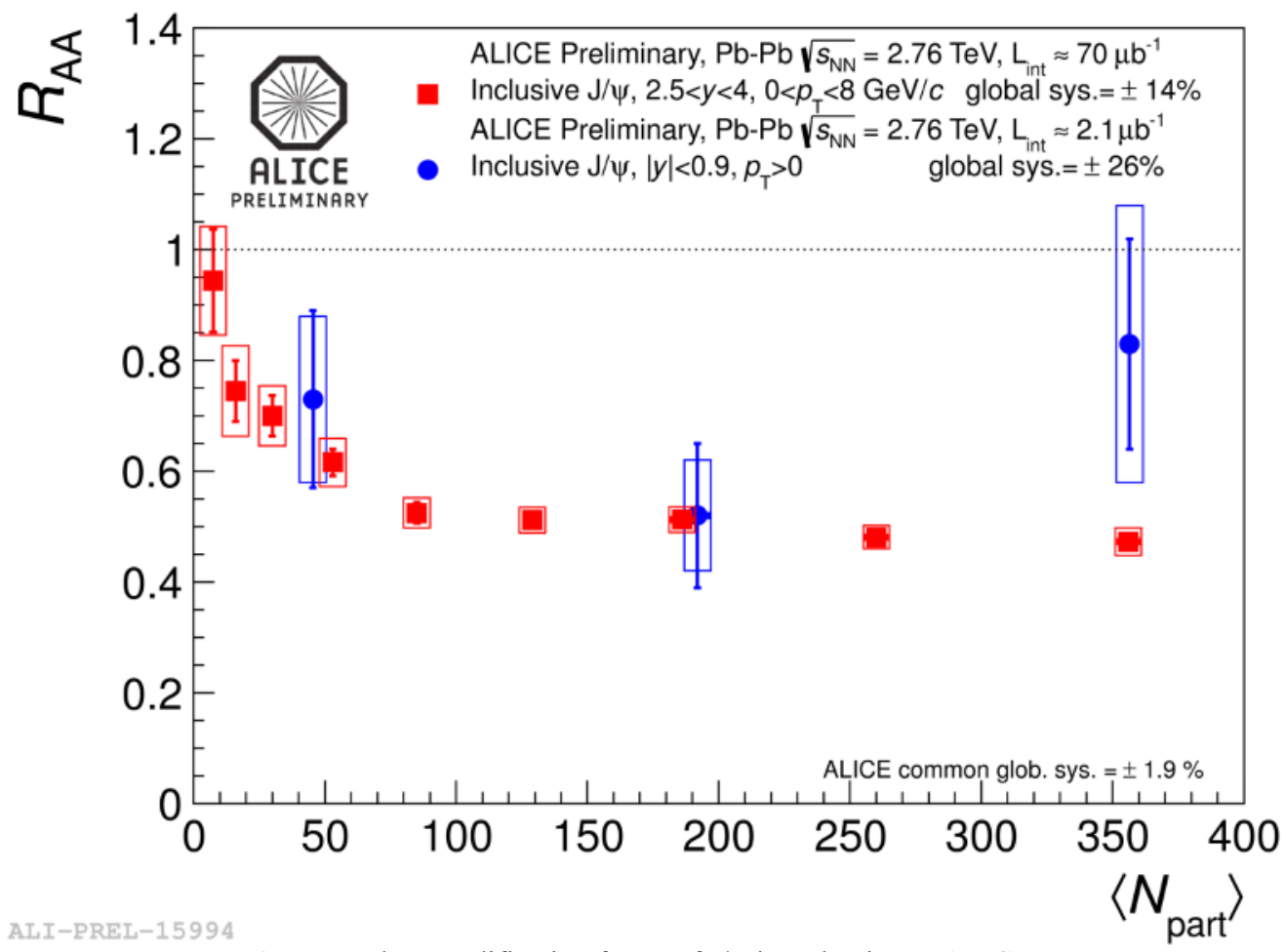

Fig. 5. Nuclear modification factor of $\mathrm{J} / \mathrm{psi}$ production at ALICE 
At LHC energies the picture for charmonium (c-cbar bound states) is different: it is produced in larger amounts, and the probability of having a c quark close enough to a cbar quark at hadronization is no longer negligible. In this case, an initial suppression of $\mathrm{J} / \mathrm{psi}$ yield (the fundamental state of charmonium) with increasing centrality followed by a saturation of suppression (or even a yield increase) is expected.

Since the different states of charmonium and bottonium are expected to melt at different temperatures, the measure of the suppression pattern of these resonances can provide a probe of the temperature reached in the QGP, telling us how close we are getting to the initial start of the Universe. The suppression can be measured by means of the nuclear modification factor $\mathrm{R}_{\mathrm{AA}}$, which givest the deviation in yields from A-A collisions relative to the scaled yields from nucleon-nucleon collisions. First ALICE data shows that the charmonium suppression is indeed saturating with the number of participants (Fig. 5), and there is a hint of regeneration in the central rapidity zone.

Bottonium suppression is harder to measure, since bottonium production cross-section is much smaller than charmonium one. ALICE is currently accumulating statistics. It has to be remember that the LHC is shared with other experiments and it runs p-p collisions for most of its duty-cycle, reserving only one month of each year to AA collisions. The foreseen LHC upgrade will help to increase the sample of events, allowing to make even more significant statements about the nature and the temperature of the medium created in the extreme conditions of temperature and energy density through which the whole Universe passed about 13.7 billions of years ago.

1. The ALICE Collaboration, J. Phys. G 30, 1517 (2004)

2. The ALICE Collaboration et al, JINST 3 (2008)

3. The ALICE Collaboration, ALICE Zero-Degree Calorimeter (ZDC) Technical Design Report, CERN-LHCC-99-005,

4. Matsui T. and Satz H., Phys. Lett. B 178416 (1986) 
